\title{
Posición de las coronas como mecanismo para tolerar el pastoreo en especies forrajeras del pastizal bajo en la región semiárida central de Argentina
}

\author{
Estelrich, H.D. ${ }^{1 @ ; ~ M a r t i n, ~ F . ~}{ }^{1}$ y Ernst, R.D. ${ }^{2}$
}

'Facultad de Agronomía. UNLPam. Argentina.

${ }^{2}$ Facultad de Ciencias Exactas y Naturales. UNLPam. Argentina.

\section{PALABRAS CLAVE ADICIONALES}

Herbivoría.

Enterrado de yemas.

Forrajeras naturales.

\section{ADDITIONAL KEYWORDS}

Herbivory.

Buried buds.

Natural forage.

\section{INFORMACIÓN}

Cronología del artículo.

Recibido/Received: 28.09.2015

Aceptado/Accepted: 22.06.2016

On-line: 15.09 .2016

Correspondencia a los autores/Contact e-mail:

estelrich@agro.unlpam.edu.ar

\section{RESUMEN}

Los pastizales naturales cubren importantes extensiones en distintas latitudes del mundo y sobre todo en áreas de clima árido y semiárido. La importancia de estos ecosistemas radica en el uso al que están destinados, que es la producción ganadera. En la región semiárida central de Argentina predominan áreas cubiertas por pastizales naturales (pastizales bajos y samófilos), bosques de Prosopis con pastizal, arbustales y matorrales con pastizal. El pastoreo es uno de los principales factores que producen cambios florísticos y estructurales en esta región donde aún existen diferentes especies que han persistido ante la acción del pastoreo. Resultados preliminares muestran diferencias en la estructura de las plantas cuando se comparan individuos pastoreados y otros provenientes de áreas clausuradas. La hipótesis de este trabajo es que las especies forrajeras que actualmente persisten son capaces de tolerar el pastoreo porque se aclimataron frente a la acción de los herbívoros, produciendo macollos con sus coronas cada vez más enterrados. El objetivo del trabajo fue evaluar en situaciones de pastoreo y de no pastoreo el diámetro de corona y la profundidad de enterrado en poblaciones de Poa ligularis, Piptochaetium napostaense, Digitaria californica y Bothriochloa springfieldii entre especies forrajeras, y en poblaciones de Jarava ichu, Amelichloa brachychaeta y Nassella tenuisima entre las no forrajeras. El trabajo se realizó en la región de colinas y lomas de la Provincia de La Pampa (Argentina) donde se establecieron transectos en áreas pastoreadas y en áreas excluidas del pastoreo. Para todas las áreas bajo pastoreo se determinó la presión de pastoreo a partir del tiempo de pastoreo y la carga animal. Los resultados obtenidos muestran mayor profundidad de enterrado de las coronas de las especies forrajeras en condiciones de pastoreo, mientras que en las especies no forrajeras como las pajas no se observan diferencias. Estos resultados podrían indicar que las especies forrajeras de los pastizales de la Región Semiárida Central de Argentina, que crecen bajo condiciones de pastoreo tienden a favorecer cierto desarrollo en profundidad de las coronas y, con ello, contribuir a la protección de los puntos de crecimientos basales de la acción del pastoreo y condiciones ambientales adversas.

\section{Grazing tolerance associated to variation in buried crown in foraging species of a short grassland in the semiarid central Argentina}

\section{SUMMARY}

Natural grasslands cover important extensions in different latitudes of the world, especially in arid and semiarid regions. The importance of these ecosystems is the use to which they are intended, which is livestock production. In the central semiarid region of Argentina, common vegetation types are natural grasslands (low and sandy grasslands), Prosopis woodlands and shrublands. Cattle grazing is one of the main factors causing floristic and structural changes in this region, where there are many grass species that are able to withstand this grazing pressure. Prior results show differences in plant structure when plants from grazed and non-grazed areas were compared. The hypothesis of this work was that forage species that persist today are acclimated to the action of herbivores, and are able to withstand grazing by producing tillers from increasingly buried crowns. Crown diameter and burial depth of forage species (Poa ligularis, Piptochaetium napostaense, Digitaria californica and Bothriochloa springfieldii) and non forage species (Jarava ichu, Amelichloa brachychaeta and Nassella tenuissima) were evaluated under grazing and non grazing conditions. The work was conducted in the region of hills and ridges of the Province of La Pampa where transects were established in grazed and non grazed areas. For all areas under grazing, livestock pressure was determined on the basis of time of grazing and stocking rate. Results show that crowns of forage plant species were buried deeper in grazed areas than in enclosures, whereas crown burial depth of non-forage species present no differences between grazing and non grazing sites. These results may indicate that forage species in grasslands of the semiarid central region of Argentina growing under grazing pressure tend to favor deeper buds development thus contributing to the protection of grazing basal growth points against adverse environmental conditions. 


\section{INTRODUCCIÓN}

Los pastizales naturales cubren importantes extensiones en distintas latitudes del mundo y sobre todo en áreas de clima árido y semiárido (Sala et al., 2013) donde la importancia de estos ecosistemas radica en el uso al que están destinados que es la producción ganadera (Nazar Anchorena, 1988). Existen diferencias entre la dinámica de los componentes del pastizal y la respuesta individual de las especies que los componen según su origen, su historia de pastoreo y el tipo de herbívoro (Milchunas et al., 1988; Diaz et al., 2007). Todo ello hace imposible hablar de modelos que generalicen el funcionamiento de estos sistemas y su respuesta al pastoreo. Cada uno de los pastizales en el mundo posee características estructurales y funcionales que les son propias haciendo que reaccionen de maneras diferentes (Diaz et al., 2001 y 2007).

En la Argentina casi el $70 \%$ de la superficie se halla cubierta por estas comunidades vegetales extendiéndose desde el norte con pastizales de altura, arbustales con pastizal, bosques xerófitos y húmedos hasta pastizales halófilos, matorrales y estepas arbustivas en la patagonia (Cabrera, 1976).

En la región semiárida central de Argentina, gran parte de la superficie se encuentra cubierta por pastizales naturales propiamente dichos (pastizales bajos y samófilos) o bosques de Prosopis con pastizal, arbustales y matorrales con pastizal (Cano et al., 1980; Cano 1988, Estelrich y Castaldo, 2014). En todos los casos estás áreas marginales a la agricultura se destinan al uso exclusivamente ganadero, ya sea cría vacuna, ovinos y caprinos (Estelrich et al., 2005).

Estos pastizales naturales se desarrollaron sin soportar grandes rebaños de herbívoros hasta la llegada de los europeos (Bucher, 1987; Deregibus, 1988) con los herbívoros domésticos y como consecuencia de ello, durante los últimos cinco siglos se produjo un marcado impacto en su dinámica y evolución (Sala et al., 1986; Deregibus, 1988, Milchunas et al., 1988; Rusch y Oesterheld, 1997; Olff y Ritchie, 1998; Chaneton et al., 2002). Por lo tanto, el pastoreo es señalado como uno de los principales factores que producen cambios florísticos y estructurales en los pastizales naturales de esta región
(Morici et al., 2003; Bertiller et al., 2004; Bisigato et al., 2005, Estelrich et al., 2005, Morici et al., 2009; Loydi y Distel, 2010).

En estas condiciones de manejo se establecen nuevas situaciones o estados de producción de los pastizales, con importantes pérdidas de superficie útil para el pastoreo, ya sea por la invasión de leñosas o el incremento de áreas cubiertas por gramíneas no forrajeras (Estelrich et al., 2005).

Las especies que componen estos sistemas responden de manera diferente a la acción de los herbívoros domésticos (Cano, 1988). Algunas de ellas, gramíneas no valiosas desde el punto de vista forrajero (Jaraba ichu, Amelichloa brachychaeta, Nassella tenuissima, N. trichotoma), incrementan notablemente su presencia en los pastizales ya que su estrategia ha sido la evasión del pastoreo por sus altos contenidos de sílice y pared celular (lignina, celulosa y hemicelulosa) (Cano, 1988, Estelrich y Cano, 1996). Por otra parte, se encuentran las especies valiosas o forrajeras (Poa ligularis, Piptochaetium napostaense, Nassella tenuis, N. longiglumis, Digitaria californica, Bothriochloa springfieldii) que si bien aún persisten en los pastizales de la región, sus estrategias para tolerar la herbivoría aún no se conocen adecuadamente.

El manejo adecuado de la carga animal en estas áreas, implica conocer los mecanismos de respuesta de las distintas especies que componen el pastizal, especialmente las de mayor valor forrajero (Morici et al., 2006). Si bien algunos resultados preliminares obtenidos en el área de pastizales bajos muestran diferencias en la estructura y tamaño de las plantas cuando se comparan individuos pastoreados y otros provenientes de áreas clausuradas (Berrueta, 1996), o también en un gradiente de distancia a la aguada (Morici et al, 2003; 2006) aún no se conocen adecuadamente los mecanismos involucrados. Para favorecer la persistencia e incrementar la abundancia de las especies forrajeras en los pastizales de la región semiárida central de Argentina, es necesario conocer mediante qué estrategia han persistido hasta la actualidad bajo condiciones de pastoreo y/o sobrepastoreo, aun cuando carecen de una historia de pastoreo importante. Posiblemente estas especies son capaces de tolerar el pastoreo porque

Tabla I. Profundidad de enterrado y diámetro promedio de las coronas de las principales especies forrajeras y no forrajeras de la región de las colinas de La Pampa en situaciones de pastoreo y en ausencia del mismo (Burial depth and average diameter of crowns of main forage and non forage species in low semiarid grasslands of central Argentina under grazing and non-grazing conditions).

\begin{tabular}{|c|c|c|c|c|c|c|}
\hline \multirow[t]{2}{*}{ Especies } & \multicolumn{3}{|c|}{ Profundidad enterrado $(\mathrm{cm})$} & \multicolumn{3}{|c|}{ Diámetro $(\mathrm{cm})$} \\
\hline & Con pastoreo & Sin pastoreo & $t$ & Con pastoreo & Sin pastoreo & $t$ \\
\hline Piptochaetium napostaense & 3,24 & 1,97 & $16.88^{*}$ & 5,24 & 6,48 & s \\
\hline Poa ligularis & 4,53 & 2,88 & $15.66^{*}$ & 4,83 & 6,24 & $s$ \\
\hline Digitaria californica & 3,18 & 1,87 & $15.98^{*}$ & 5,50 & 5,54 & ns \\
\hline Botrhiochloa springfieldii & 3,51 & 1,98 & $12.03^{*}$ & 6,25 & 7,27 & s \\
\hline $\begin{array}{l}\text { Pajas (Nassella ichu, N. tenuissima, } \\
\text { Amelichloa brachychaeta) }\end{array}$ & 3,77 & 3,88 & $0.46 \mathrm{~ns}$ & 6,83 & 6,97 & ns \\
\hline
\end{tabular}

*: diferencias significativas entre medias en situaciones de pastoreo y en ausencia del mismo, ns: diferencias no significativas (test de Student, $p<0,05)$ (significant differences between means from grazing and non grazing situation, ns: no significant difference (Student's test, $p<0,05)$. 
dispondrían de alguna estrategia morfofisiológica relacionada con el tamaño de las coronas y la profundidad de enterrado de los individuos que puede variar de acuerdo con la presión de pastoreo.

En los trabajos que describen la biología y dinámica de las gramíneas en condiciones de pastoreo (Briske, 1991; Briske y Derner, 1998, Briske et al., 2011), la profundidad de enterrado de las coronas de las plantas no aparece como un rasgo de las especies relacionado con la acción de los herbívoros. Posiblemente esto se debe a que la mayor parte de los estudios se han realizado en pastizales con larga historia de herbivoría, donde este mecanismo ya podría estar incorporado entre las estrategias de las especies para tolerar el pastoreo. La importancia de estudiar las coronas en este trabajo radica en que albergan los puntos de crecimiento intercalares, axilares y apicales y también son capaces de acumular reservas en forma de hidratos de carbono. Todo esto hace que las coronas tengan un papel preponderante en la restitución del área foliar de las gramíneas y la perennidad de las especies forrajeras luego de ser sometidas al pastoreo (Briske et al., 2011). Las diferencias observadas en la arquitectura de las plantas forrajeras pastoreadas y las no pastoreadas donde la profundidad de enterrado de las coronas es evaluada como una variable relacionada con el pastoreo (Berrueta, 1996; Morici et al., 2003; 2006), aún no se ha estudiado como un posible mecanismo para tolerar el mismo.

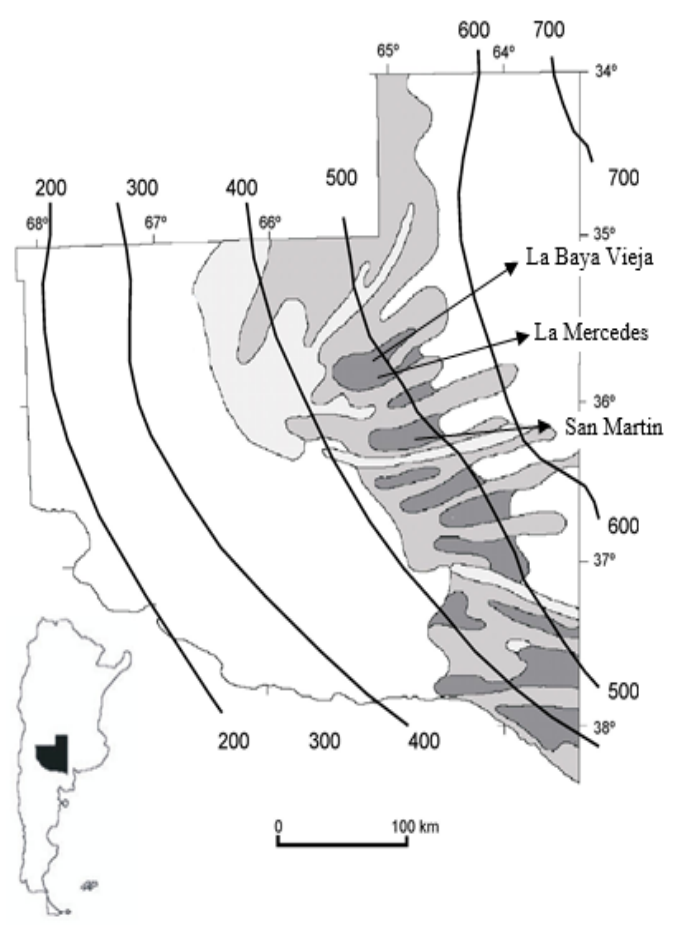

Figura 1. Ubicación relativa de los muestreos en el área de Pastizales Bajos de la provincia de La Pampa (gris claro: Pastizal samófilo; gris intermedio: Bosque de caldén; gris oscuro: Pastizal bajo). Lugares de muestreo: establecimientos La Baya Vieja, La Mercedes y San Martin (Relative location of the sampling area in the low semiarid grasslands of central Argentina (light gray: sandy grassland; medium gray: calden forest; dark gray: low grassland).
El objetivo del presente trabajo fue evaluar en situaciones de pastoreo y de no pastoreo, el diámetro de corona y la profundidad de enterrado de las coronas en poblaciones de especies forrajeras de Poa ligularis, de Piptochaetium napostaense, de Digitaria californica, de Bothriochloa springfieldii, y en poblaciones de Jarava ichu, de Amelichloa brachychaetay de Nassella tenuissima entre las no forrajeras.

\section{MATERIALES Y METODOS}

El trabajo se realizó en tres establecimientos ganaderos de cría y eventualmente recría, ubicados en la Provincia de La Pampa (Argentina) región fisiográfica Oriental, subregión de colinas y lomas. (Cano et al., 1980; figura 1). Las precipitaciones medias anuales oscilan entre 500-600 mm concentradas principalmente en verano y otoño (Casagrande y Conti, 1980; figura 2) y el relieve es de ondulado a colinado (Salazar Lea Plaza 1980). Los suelos predominantes son Haplustoles enticos y Ustisamentes típicos (Peña Zubiate et al., 1980).

En la región predominan pastizales de gramíneas bajas (Piptochaetium napostaense y Poa ligularis entre las de invierno, y Digitaria californica, Bothriochloa springfieldii entre las de verano) e intermedias (Jarava ichu, Nassella tenuissima, N. trichotoma, Amelichloa brachychaeta y Aristida niederleinii), con arbustos bajos (Lycium chilense, L. gilliesianum, Aloysia gratissima). Se observa también en forma dispersa un estrato arbóreo y arbustivo bajo, se encuentran Prosopis caldenia y Condalia microphylla (Cano, 1988).

Para el muestreo se seleccionaron tres establecimientos con predominancia de pastizal bajo (Establecimientos La Mercedes, La Baya Vieja y San Martin, figura 1). En cada uno de ellos se seleccionaron dos áreas de pastizal bajo en condiciones de pastoreo y en ausencia del mismo (calles, banquinas, clausuras). En cada área se establecieron 2 transectos de $200 \mathrm{~m}$ de longitud, y en cada uno de ellos se ubicaron estaciones de muestreo cada $10 \mathrm{~m}$. En cada transecto se extrajeron 20 individuos de cada una de las

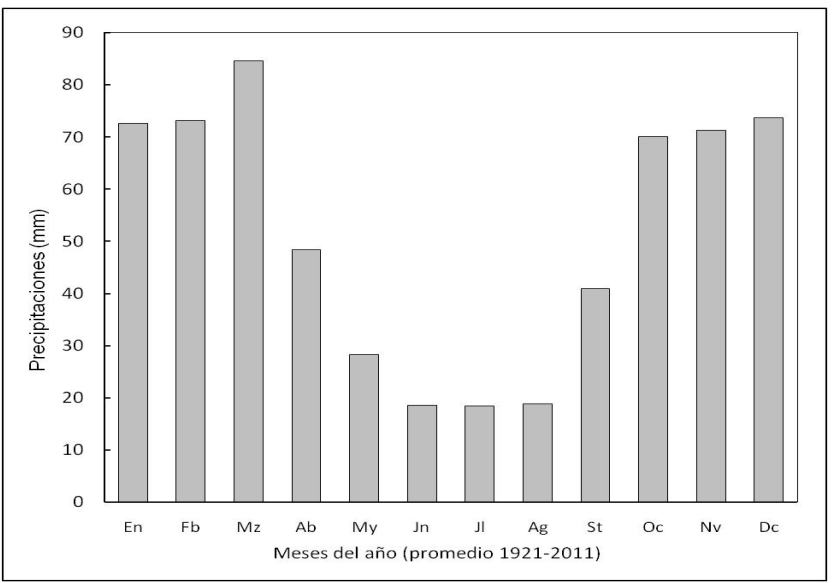

Figura 2. Distribución de las precipitaciones promedio mensuales para la ciudad de Toay $(20 \mathrm{~km}$ del área de muestreo; promedio del período 1921-2011, Fuente: Administración Provincial del Agua, Gobierno de la provincia de La Pampa) (Distribution of monthly average rainfall for the city of Toay (20 km from sampling area; average period from 1921 to 2011. Source: Provincial Water Management, Government of the province of La Pampa). 


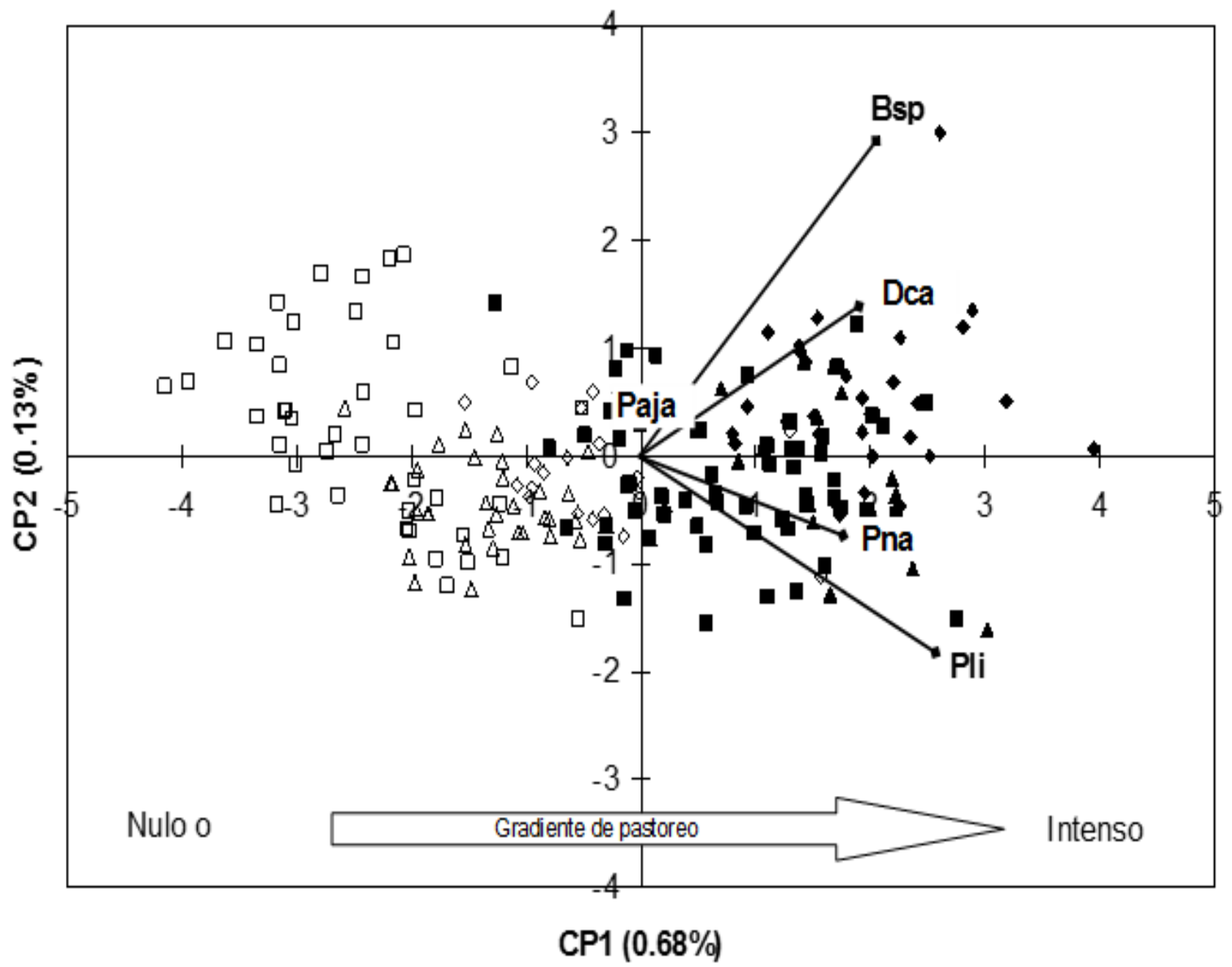

Figura 3. Análisis de Componentes principales (ACP) de la profundidad de enterrado de las coronas de las distintas especies (Pli: Poa ligularis; Pna: Piptochaetium napostaense; Dca: Digitaria californica; Bsp: Botrhiochloa springfieldii y gramíneas no forrajeras: Nassella ichu; N. tenuissima; Amelichloa brachychaeta) en condiciones de pastoreo y en ausencia del mismo en pastizales de la región de las colinas de La Pampa. Símbolos rellenos: situación de pastoreo, símbolos vacíos: ausencia de pastoreo (Principal component analysis (PCA) of crowns burial depth of the different plant species (Pli: Poa ligularis; Pna: Piptochaetium napostaense; Dca: Digitaria californica; Bsp: Botrhiochloa springfieldii and non-forage species: Nassella ichu; N. tenuissima; Amelichloa brachychaeta) under grazing and non-grazing conditions in grasslands of the hills region of La Pampa. Filled symbols: grazing situation; empty symbols: no grazing).

especies forrajeras (Poa ligularis, Piptochaetium napostaense, Digitaria californica, Bothriochloa springfieldii) y no forrajeras (Jarava ichu, Amelichloa brachychaeta y Nassella tenuissima). Estas especies son perennes y de acuerdo a la posición de sus yemas de renuevo a ras del suelo, son consideradas hemicriptofitas (Raunkiaer, 1934). Para cada individuo extraído se registró el diámetro de la corona y la profundidad de enterrado (distancia existente entre la base de los macollos desde donde nacen las raíces y el lugar donde las hojas emergen a la superficie del suelo).

Estas áreas con pastizales naturales son utilizadas para la cría de ganado bovino y la raza utilizada en la región es Angus. Para cada uno de los establecimientos en los que se realizó el muestreo, se registró la carga ganadera y el tipo de pastoreo al que ha sido sometido cada potrero en los últimos años. El pastoreo se realiza generalmente durante otoño, invierno y principio de primavera y depende del manejo de cada establecimiento como se distribuye la carga en cada potrero durante el año. Por ejemplo, en un potrero donde se realizó el muestreo para este trabajo, el pastoreo se realizó con 4 bovinos.ha-1 ${ }^{-1}$ durante 40 días en el otoño o el otro extremo, 0,6 bovinos $\cdot$ ha $^{-1}$ durante todo el año. Así a través de la carga y el periodo de permanencia de los animales (bovinos de entre 350 y $400 \mathrm{~kg}$ de peso vivo), se estimó la presión de pastoreo para cada potrero como un índice que toma valores de $0-0,7$ bovinos $\cdot \mathrm{ha}^{-1}$. año-1.

Se realizo un primer estudio exploratorio mediante análisis de componentes principales (ACP), las diferencias entre medias de profundidad de enterrado y diámetro de corona de plantas provenientes de áreas pastoreadas y no pastoreas se establecieron mediante test de Student $(p<0,05)$. Las relaciones entre variables se analizaron mediante regresión y correlación (Di Renzo et al., 2014).

\section{RESULTADOS Y DISCUSIÓN}

A partir de un análisis exploratorio de los resultados obtenidos se pudo diferenciar la profundidad de enterrado de las especies forrajeras en áreas con y sin pastoreo. En la figura 3 se muestran los resultados obtenidos del ACP donde se comparan todas las obser- 


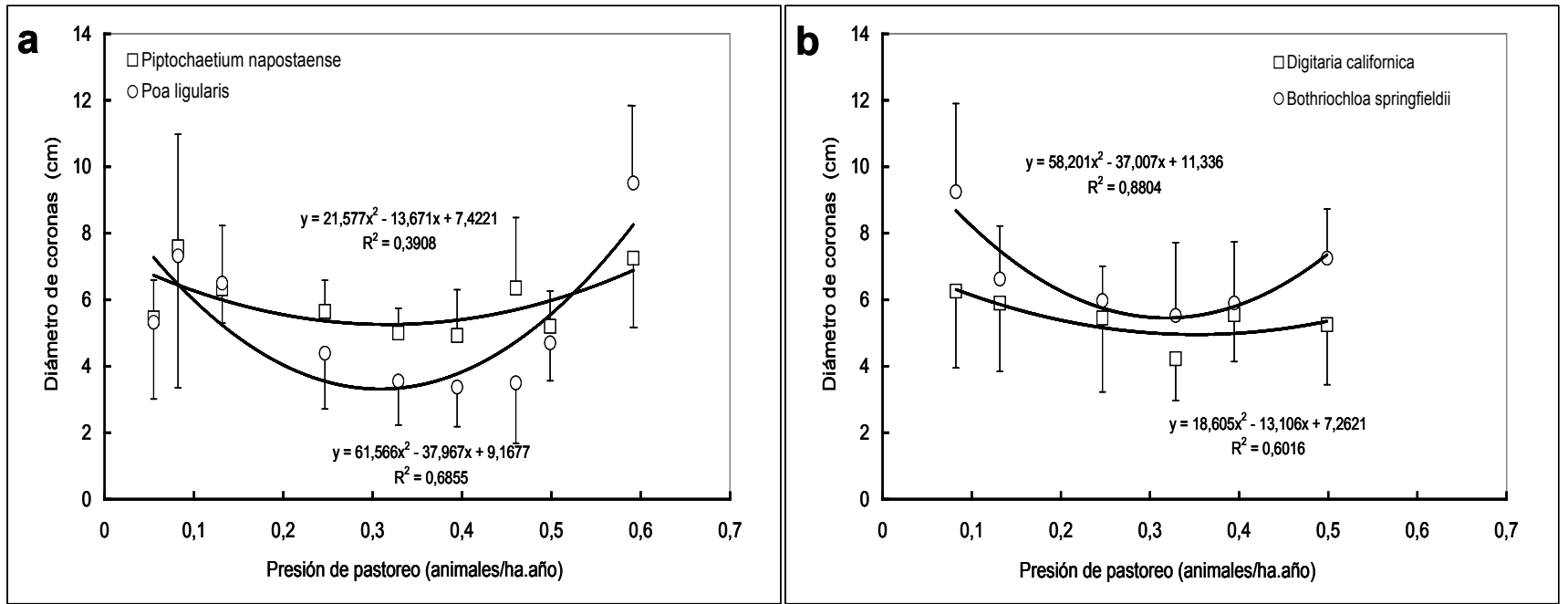

Figura 4. Relación entre el diámetro de las coronas $(\mathrm{cm})$ y la presión de pastoreo (animales $\cdot \mathrm{ha}^{-1} \cdot \mathrm{año}^{-1}$ ) para (a) Poa ligularis y Piptochaetium napostaense, (b) Digitaria californica y Bothriochloa springfieldii en pastizales de la región semiárida central de Argentina (Relation between the crown diameter $(\mathrm{cm})$ and grazing pressure (number of animals ha1.año-1) for (a) Poa ligularis and Piptochaetium napostaense, (b) Bothriochloa springfieldii and Digitaria californica in low semiarid grasslands of Argentina).

vaciones realizadas en las diferentes áreas de muestreo y para las diferentes especies. El eje 1 tiene un $68 \%$ de explicación de la distribución de los muestreos y representa un gradiente en el que se ordenan claramente las áreas sin y con pastoreo, mientras que el eje 2 explica en un $13 \%$ qué especies tienen diferencias de enterrado a lo largo ese gradiente de pastoreo. Se observa que todas las especies de gramíneas no forrajeras, no se diferencian con el eje 2, mientras que las especies forrajeras aparecen bien diferenciadas entre las que se destacan Botrhiochloa springfieldii y Poa ligularis las que son más susceptibles a enterrarse frente al pastoreo.

En la tabla I se muestran los promedios de profundidad de enterrado y del tamaño de las coronas de las principales especies forrajeras y no forrajeras en situaciones de pastoreo y en ausencia del mismo. En todas las especies forrajeras las coronas estuvieron más enterradas en situaciones de pastoreo $(\mathrm{p}<0,05)$. Con respecto al tamaño de las coronas, la mayoría de las especies forrajeras poseen menores diámetros en situaciones de pastoreo $(p<0,05)$, mientras que en Digitaria californica, no se observan diferencias con las situaciones de ausencia de herbívoros $(p>0,05)$.

De todas las especies estudiadas, Poa ligularis, una de las principales forrajeras de los pastizales de la región semiárida, presenta las mayores diferencias en la profundidad de enterrado de sus coronas, 4,53 y 2,88 $\mathrm{cm}$ en situaciones de pastoreo y ausencia del mismo respectivamente $(\mathrm{p}<0,05)$.

En tanto para Piptochaetium napostaense, otra especie típica de los pastizales de esta región, si bien se observa que en condiciones de pastoreo sus coronas están enterradas a mayor profundidad que en condiciones de no
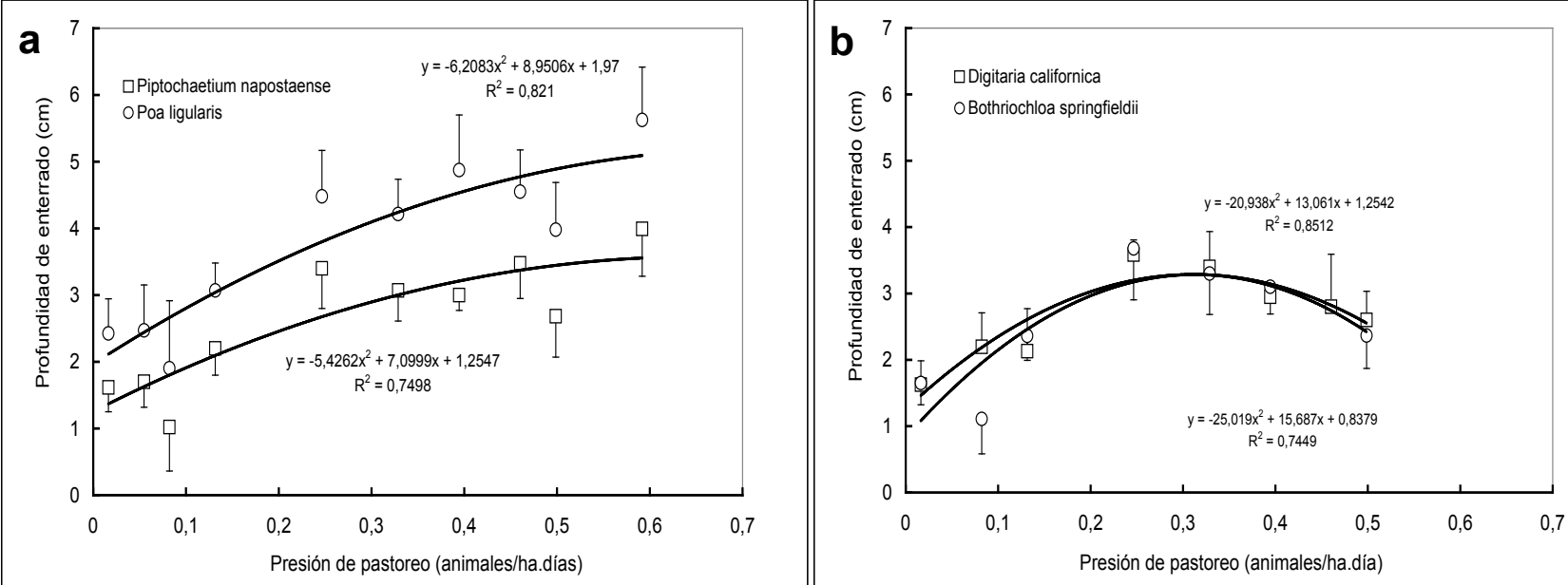

Figura 5. Relación entre la profundidad de enterrado de las coronas $(\mathrm{cm})$ y la presión de pastoreo (animales·ha${ }^{1}$-año-1) para (a) Poa ligularis y Piptochaetium napostaense, (b) Digitaria californica y Bothriochloa springfieldii en pastizales de la región semiárida central de Argentina (Relation between plant crown burial depth $(\mathrm{cm})$ and grazing pressure (number of animals $\cdot$ ha $^{-1} \cdot$ año-1 $^{-1}$ for (a) Poa ligularis and Piptochaetium napostaense, (b) Bothriochloa springfieldii and Digitaria californica in low semiarid grasslands of central Argentina). 


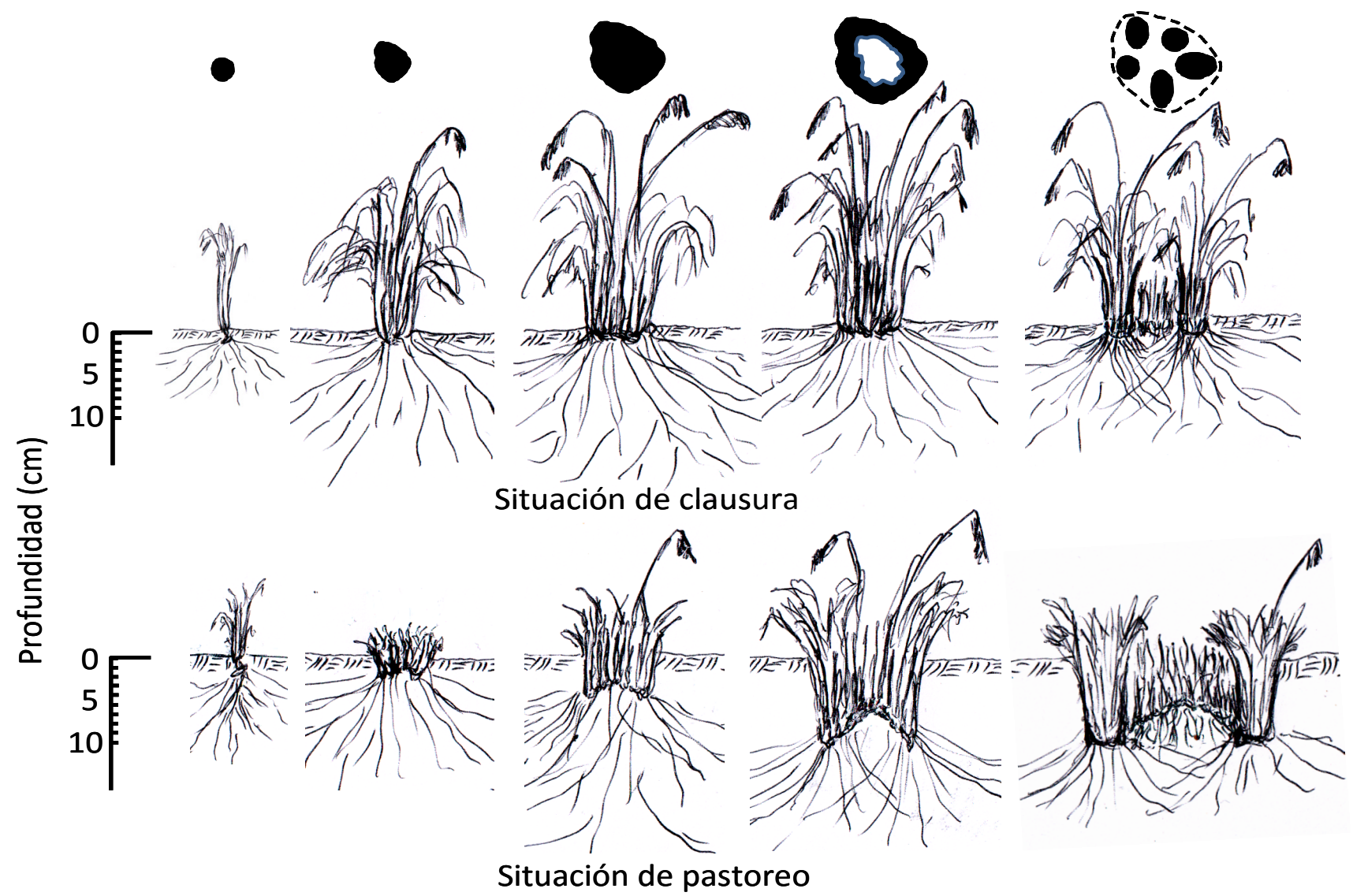

Figura 6. Modelo conceptual de la dinámica de la estructura de las plantas en situaciones de pastoreo y de clausura (adaptado de Briske y Derner, 1998) (Conceptual model of vegetation structure dynamics under grazing and non grazing conditions (adapted from Briske y Derner, 1998).

pastoreo $(\mathrm{p}<0,05)$ los valores registrados de 3,24 y 1,97 $\mathrm{cm}$ respectivamente, son inferiores a los de Poa ligularis. En cuanto a las especies estivales Digitaria californica y Bothriochloa springfieldii aun cuando se detectaron diferencias en la profundidad de enterrado $(p<0,05)$ de sus coronas entre áreas con y sin pastoreo, estas no fueron tan importantes como en el caso de las especies invernales mencionadas precedentemente. Por último, las especies no forrajeras, no muestran diferencias en la profundidad de enterrado o el tamaño de sus coronas con 3,77 - 3,88 cm y 6,83-6,97 cm respectivamente, en áreas con y sin pastoreo $(\mathrm{p}>0,05)$.

En todos los casos se puede observar una relación entre el diámetro y la profundidad de enterrado de las coronas con la presión de pastoreo (figuras 4 y 5). En Poa ligularis y en Botrhriochloa springfieldii el diámetro de corona es muy afectado por la presión de pastoreo $\left(R^{2}=0,69\right.$ y 0,88 respectivamente, figura $4 \mathbf{a}$ y $\left.4 \mathbf{b}\right)$ mientras que en Piptochaetium napostaense y en Digitaria californica este efecto es menos evidente $\left(R^{2}=0.39\right.$ y 0.60 respectivamente; figura $\mathbf{4 a}$ y $\mathbf{4 b}$ ). En las especies de invierno con presiones intermedias de pastoreo las coronas tienden a ser más pequeñas y se observa menor variabilidad en el tamaño de las mismas (figura 4a).

Con respecto a la profundidad de enterrado, en las especies de invierno se observa una tendencia creciente conforme aumenta la presión de pastoreo $\left(\mathrm{R}^{2}=\right.$ 0,82 y 0,75 para Poa ligularis y Piptochaetium napostaense respectivamente; figura 5 a). Los menores valores de enterrado 1,6 y $2,5 \mathrm{~cm}$ para Piptochaetium napostaense y Poa ligularis coincidieron con las menores presiones de pastoreo o con las situaciones de clausura, mientras que los mayores valores de enterrado oscilaron en 4 y $5,8 \mathrm{~cm}$ respectivamente y coincidieron con las mayores presiones de pastoreo (figura 5a).

En las especies que vegetan en verano se observa un comportamiento similar hasta presiones de pastoreo intermedias, donde las coronas aumentan su profundidad de enterrado hasta un momento a partir del cual se mantienen o comienzan a disminuir su profundidad con las mayores presiones de pastoreo $\left(R^{2}=0,74\right.$ y 0,85 para Digitaria californica y Bothriochloa springfieldii respectivamente, figura $5 \mathbf{b}$ ).

Esto podría explicarse debido al tipo de manejo que se realiza en la región, donde la mayor presión de pastoreo se realiza durante los meses de otoño-invierno por la inclusión del pasto llorón (Eragrostis curvula) en la cadena forrajera. Por ello estas especies podrían responder enterrando sus coronas hasta una cierta presión de pastoreo y luego cuando esta se incrementa, la profundidad de enterrado disminuye porque el pastoreo intenso y continuo reduce la posibilidad de acumulación y/o reposición de reservas.

Estos resultados coinciden con lo reportado por la bibliografía respecto al efecto homogeneizador del pastoreo a presiones intermedias (Sala et al., 1986). Mientras que en los extremos del gradiente de pastoreo se 
pondrían en evidencia otros procesos fisiológicos que afectarían el desarrollo y tamaño de las matas (Briske 1991, 1996).

Cuando el pastoreo es leve o nulo los macollos florecen y mueren de acuerdo con las jerarquías de aparición, los más viejos del centro de la mata son los que florecen y mueren primero (Briske y Derner, 1998; figura 6). Cuando el pastoreo es continuo e intenso, las matas son pastoreadas y sobrepastoreadas, visitadas más de una vez por los herbívoros que consumen permanentemente sus rebrotes con importantes consecuencias en la asignación de recursos en las plantas (Briske y Richards, 1995; Briske 1996; Derner et al., 2006). Frente a esta situación las matas tienden a no florecer y se mantienen en un estado de macollaje permanente por ruptura de dominancia apical (Murphy y Briske, 1992; Briske et al., 2011) y mayor llegada de luz a los puntos de crecimiento, con lo que incrementa el tamaño de las matas en cuanto a su diámetro. Por otra parte, este mismo proceso llevaría a un agotamiento de reservas de los macollos más viejos y menos enterrados y en consecuencia la muerte de los mismos (figura 6). A su vez, los macollos que mueren en el centro de las plantas podrían restituir parte del carbono orgánico y nutrientes necesarios para la persistencia y desarrollo de las nuevas plantas provenientes de la fragmentación de matas de mayor tamaño (Briske y Derner, 1998; Briske et al., 2011).

La degradación de las matas de las especies forrajeras (en especial Poa ligularis y de Piptochaetium napostaense) de los pastizales de esta región en condiciones de pastoreo, no sólo podría explicarse por la reducción en la actividad fotosintética y/o tejidos meristemáticos necesarios para el crecimiento de las plantas, sino también por una disminución en el ingreso de materia orgánica y nutrientes en los suelos debajo de las matas y en las inmediaciones de las mismas (Briske y Derner, 1998; Briske et al., 2011). Este proceso se caracteriza entonces por una reducción en el área basal de las plantas y un aumento en la densidad de las mismas, muy probablemente como resultado dela fragmentación de grandes matas individuales (Briske y Derner, 1998; figura 6). Luego de la fragmentación de las matas de grandes diámetros, a partir de los clones más jóvenes se conforman varias matas independientes de diámetros más pequeños y con las coronas más enterradas. Esto último puede ser producto de modificaciones de la arquitectura y dinámica del sistema subterráneo de las plantas, inducido por el pastoreo y por el cual también incrementan sus reservas (Derner et al., 2006; figura 6).

La mayor profundidad de enterrado de las coronas que se observa en las plantas forrajeras en situaciones de pastoreo, podría intentar explicarse por la acción erosiva del pisoteo del ganado y la acumulación de material edáfico alrededor de las matas, con lo cual el mecanismo de enterrado de coronas y protección de los puntos de crecimiento no sería tal. Si ello fuera así, entonces la profundidad de enterrado de las pajas también debería ser afectada de igual manera y sin embargo ello no fue observado (tabla I).

Otra posibilidad para explicar esta diferencia de enterrado de las coronas de las especies forrajeras sería el establecimiento de las plántulas a diferente profundidad en el suelo y que posteriormente el ganado en condiciones de pastoreo sea el responsable de arrancar aquellos individuos menos enterrados. Esta situación no sería sustentada por la dinámica de establecimiento de las especies en condiciones naturales, donde por un lado, las semillas de Piptochaetium napostaense se auto entierran a profundidad similar que va de 1 a $2 \mathrm{~cm}$ (Ernst et al., 2011) y por otro lado, las de Poa ligularis, Botrhriochloa springfieldii y Digitaria californica son depositadas sobre el suelo o la broza (Ernst et al., 2011), desde donde se inicia el proceso de germinación cuando las condiciones ambientales son adecuadas. Es decir que todas las especies germinarían sus semillas desde la superficie y no habría posibilidades de un establecimiento diferencial de los individuos tan marcado que diferencie posteriormente plantas con enterrado de coronas hasta $7 \mathrm{~cm}$ como los encontrados en el presente trabajo.

\section{CONCLUSIONES}

La mayor profundidad de enterrado que se observa en las coronas de plantas forrajeras en condiciones de pastoreo en esta región, sería consecuencia de un proceso gradual y posterior al establecimiento de las plantas donde seguramente la presencia del ganado es un factor explicativo de importancia. En condiciones de pastoreo, los nuevos macollos que vayan incorporándose en la periferia de las coronas, lo harían gradualmente a mayor profundidad y con el correr del tiempo, daría como resultado plantas con las coronas más enterradas. En estas condiciones, los puntos de crecimiento resultarían más protegidos de la acción del pastoreo, y a su vez se lograría una mayor cantidad de reservas que podrían favorecer la persistencia de las plantas en condiciones de pastoreo, en especial cuando las matas se dividen y quedan clones de diámetros pequeños. El conocimiento de la fisiología de los cambios morfológicos observados podría brindar nuevos elementos para profundizar en la temática. Si bien estos resultados son preliminares, se podría pensar en este parámetro como indicador de grado de uso de algunas de las especies forrajeras de los pastizales de la región semiárida central de Argentina.

\section{AGRADECIMIENTOS}

A Julio Zapico, propietario del Est. La Mercedes, a Tomas Berongharay propietario del Est. La Baya Vieja y a Eduardo Elizondo encargado del Est. San Martin, por su hospitalidad. A Bedulia Coronado, Jorge Orias Solis y Edwin Portal Rivera nuestro agradecimiento por la ayuda brindada durante los trabajos a campo. A Francisco Babinec y Valeria Belmonte por su asesoramiento en cuestiones de estadística y análisis de datos. El trabajo fue realizado en el marco de un proyecto financiado por la Facultad de Agronomía de la UNLPam.

\section{BIBLIOGRAFÍA}

Bertiller, M.B.; Bisigato, A.J.; Carrera, A.C. y Del Valle, H.F. 2004. Estructura de la vegetación y funcionamiento de los ecosistemas del Monte chubutense. Bol Soc Arg Bot, 39: 139-158.

Berrueta, M.A. 1996. Diferencias estructurales producidos por pastoreo y no pastoreo de grandes herbívoros en una comunidad de pastizal bajo 
en la región semiárida pampeana. Beca de iniciación a la Investigación Facultad de Agronomía UNLPam.

Bisigato, A.; Bertiller, M.; Ares, J. and Pazos, E. 2005. Effects of grazing on plant patterns in arid ecosystems of the Patagonian Monte. Ecography, 28: 561-572.

Briske, D.D. 1991. Developmental morphology and physiology of grasses. In: Grazing Management: an ecological perspective. Heitschmitdt, R.K. and Stuth, J.W. (eds.). Tiumber Press. Portland. Oregon. USA. pp. 11-26.

Briske, D.D. and Richards, J.H. 1995. Plant responses to defoliation: A physiological, morphological, and demographic evaluation. In: Wildland Plants: Physiological ecology and developmental morphology. Bedunah, D.J. and Sosebee, R.E. (eds.). Society for Range Management. Denver. Colorado. pp. 635-710.

Briske, D.D. 1996. Strategies of plant survival in grazed systems: a functional interpretation. In: The ecology and Management of Grazing Systems. Hordgson J. and Illius, A.W. (eds.). CAB International, UK. pp. 37-66.

Briske, D.D. and Derner, J.D. 1998 .Clonal biology of caespitose grasses. In: Population biology of grasses. Ed. Cheplick G.P. Cambridge University Press. Nueva York. pp 106-135.

Briske, D.D.; Derner, J.D.; Milchunas, D.G. and Tate, K.W. 2011. An evidence-based assessment of prescribed grazing practices. In: Conservation Benefits of Rangeland Practices: Assessment, Recommendations, and Knowledge Gaps. Briske D.D. (ed). Allen Press, Lawrence KS. pp. 22-74.

Bucher, E.H. 1987. Herbivory in arid and semiarid regions of Argentina. Rev Chil Hist Natural, 60: 265-273.

Cabrera, A. 1976. Regiones fitogeográficas argentinas. Enc Arg Agr y Jard. Tomo II, Fasc. 2. Acmé. Buenos Aires.

Cano, E.; Fernandez, B. y Montes, M. 1980. La Vegetación de la Provincia de La Pampa y Carta de vegetación 1:500000. En: Inventario Integrado de los Recursos Naturales de la Provincia de La Pampa. Instituto Nacional de Tecnología Agropecuaria (INTA), Provincia de La Pampa, Universidad Nacional de La Pampa (UNLPam) (Eds). Buenos Aires. 493 pp. https:// recursosnaturales.lapampa.edu.ar/

Cano, E. 1988. Pastizales naturales de La Pampa. Tomo I: Descripción de las especies más importantes. Convenio AACREA - Provincia de La Pampa. 425 pp.

Casagrande, G. y Conti, H.A. 1980. Clima de la Provincia de La Pampa. En: Inventario Integrado de los Recursos Naturales de la Provincia de La Pampa. Instituto Nacional de Tecnología Agropecuaria (INTA), Provincia de La Pampa, Universidad Nacional de La Pampa (UNLPam) (Eds). Buenos Aires. 493 pp. https://recursosnaturales.lapampa.edu.ar/

Chaneton, E.J.; Perelman, S.B.; Omacini, M. and Leon, R.J.C. 2002. Grazing, environmental heterogeneity, and alien plant invasions in temperate pampa grasslands. Biol Inv, 4: 7-24.

Deregibus, V.A. 1988. Importancia de los pastizales naturales en la República Argentina: situación presente y futura. Rev Arg Prod Anim. 8: 67-78.

Derner, J.D.; Boutton, T.W. and Briske, D.D. 2006. Grazing and ecosystem carbon storage in the North American Great Plains. Plant and Soil, 280: 77-90.

Di Rienzo, J.A.; Casanoves, F.; Balzarini, M.G.; Gonzalez, L.; tablada, M.y Robledo, C.W. InfoStat versión 2014. Grupo InfoStat, FCA, Universidad Nacional de Córdoba, Argentina. URL http://www.infostat.com.ar

Diaz, S.; Noy-Meir, I. and Cabido, M. 2001. Can grazing response of herbaceus plant be predicted from simple vegetative traits?. J Applied Ecol, 38: 497-508.

Diaz, S.; Lavorel, S.; Mcintyre, S.; Falczuk, V.; Casanoves, F.; Milchunas, D.; Skarpe, C.; Rusch, G.; Stemberg, M.; Noy-Meur, I.; Landsberg, J.; Zhang, W.; Clark, H. and Campbell, B.D. 2007. Plant trait responses to grazing - a global synthesis. Global Change Biology, 13: 313-341.

Ernst, R.; Morici, E.; Muiño, W.; Berrueta, M.A. and Lerner, P. 2011. Effect of burning on the germinable seed bank. Estelrich, H.D. y Cano, A.E. 1996. Dinámica de la degradabilidad ruminal in saco de la fitomasa aérea de especies nativas de la región semiárida pampeana (Argentina). Rev Fac Agron, UNLPAM, 9: 2-16.

Estelrich, H.D.; Chirino C.C.; Morici, E.F. y Fernandez, B. 2005. Dinámica deáreas naturales cubiertas por bosquey pastizal en la región semiárida central de Argentina - Modelo Conceptual. En: Heterogeneidad de la vegetación. Libro homenaje a Rolando Leon (Paruelo J.; Oesterheld, M. y Aguiar, M. Eds.).Editorial de la Facultad de Agronomía (UBA). Buenos Aires. Argentina. $430 \mathrm{pp}$.

Estelrich, H.D. y Castaldo, A. 2014. Receptividad y carga ganadera en distintas micro regiones de la provincia de La Pampa (Argentina) y su relación con las precipitaciones. SEMIÁRIDA Rev Fac Agron, UNLPAM, 24: 7-19.

Loydi, A. y Distel, R. 2010. Diversidad florística bajo diferentes intensidades de pastoreo por grandes herbívoros en pastizales serranos del sistema Ventania, Buenos Aires. Ecol Austral, 20: 281-291.

Milchunas, D.G.; Sala, O.E. and Lavenroth, W.K. 1988. A generalized model of the effects of grazing by large herbivores on grassland community structure. Am Nat, 132: 87-106.

Morici, E.F.; Ernst, R.D.; Kin, A.; Estelrich, D.; Mazola, M. y Poey, S. 2003. Efecto del pastoreo en un pastizal semiárido de Argentina según la distancia a la aguada. Arch Zootec, 52: 59-66.

Morici, E.F.; Kin, A.; Mazola, M.; Ernst, R.D. y Poey, S. 2006. Efecto del pastoreo sobre las gramíneas perennes Piptochaetium napostaense y Poa ligularis en relación con la distancia a la aguada. Rev Fac Agron, UNLPam, 17: 1-13.

Morici, E.; Doménech García, V.; Gómez Castro, G.; Kin, A.G.; Saenz, A. y Rabotnikof, C. 2009. Diferencias estructurales entre parches de pastizal del caldenal y su influencia sobre el banco de semillas, en la provincia de La Pampa, Argentina. Agrociencia, 43: 529-537.

Murphy, J.S. and Briske, D.D. 1992. Regulation of tillering by apical dominance: Chronology, interpretative value, and current perspectives. J Range Manage, 45: 419-430.

Nazar Anchorena, J.B. 1988. Manejo de pastizales naturales de La Pampa. Tomo II. Convenio AACREA - Provincia de La Pampa. 425 pp.

Olff, H. and Ritchie, M.E. 1998. Effects of herbivores on grassland plant diversity. Tree, 13: 261-265.

Peña Zubiate, C.A.; Maldonado Pinedo, D.; Martínez, H. y Hevia, R. 1980. Suelos de la Provincia de La Pampa y carta de suelos 1:500000. En: Inventario integrado de los recursos naturales de la provincia de La Pampa. Instituto Nacional de Tecnología Agropecuaria (INTA), Provincia de La Pampa, Universidad Nacional de La Pampa (UNLPam) (Eds). Buenos Aires. 493 pp. hitps://recursosnaturales.lapampa.edu.ar/.

Raunkiaer, C. 1934. The life of plants and statistical plant geography. Oxford University Press. Oxford. 632 pp.

Rusch, G.M. and Oesterheld, M. 1997. Relationship between productivity, and species and functional groups diversity in grazed and non-grazed Pampas grasslands. Oikos, 78: 519-526.

Sala, O.E.; Oesterheld, M.; Leon, R.J.C. and Soriano, A. 1986. Grazing effects upon plant community structure in subhumid grasslands of Argentina. Vegetatio, 67: 27-32.

Sala, O. E., Vivanco, L. and Flombaum, P. 2013. Grassland Ecosystems. Vol 4 Pages 1-7. In: Levin, S.A. Editor. Encyclopedia of Biodiversity (Second Edition). Academic Press.

Salazar Lea Plaza, J.C. 1980. Geomorfología de la Provincia de La Pampa. En: Inventario integrado de los recursos naturales de la provincia de La Pampa. Instituto Nacional de Tecnología Agropecuaria (INTA), Provincia de La Pampa, Universidad Nacional de La Pampa (UNLPam) (Eds). Buenos Aires. 493 pp. https://recursosnaturales. lapampa.edu.ar/ 